\title{
PATTERN SIMILARITY IN BIOLOGIAL, LINGUISTIC, AND SOCIOCULTURAL EVOLUTION
}

\author{
NATHALIE GONTIER ${ }^{* 1}$ \\ *Corresponding Author: nlgontier@fc.ul.pt \\ ${ }^{1}$ Applied Evolutionary Epistemology Lab, Centre for Philosophy of Science, Faculty of \\ Science, University of Lisbon, Portugal
}

\begin{abstract}
Linguistic and sociocultural evolution models share pattern similarity with biological evolution models. Examples of similar patterns include descent with modification, reticulation, drift, gradualism, punctuated equilibria, cyclicity, and periodicity. Pattern similarity is often explained by assuming that the same evolutionary mechanisms are causally responsible for the patterns. We demonstrate that this argument is not always warranted because similar patterns can be induced by different mechanisms and processes. We investigate the implications this finding has on how we define mechanisms and on how biological, sociocultural and linguistic evolution relate and diverge from one another.
\end{abstract}

\section{Introduction}

Scholars are currently applying a similar set of macro-oriented phylogenetic methods (Pagel, 1999) and micro-oriented experimental evolution techniques (Mesoudi, 2016; Tamariz \& Kirby 2016) or computer-generated simulations to model and mimic biological, linguistic and sociocultural evolution (Steels, 2015). Results of research indicate that biological, linguistic and cultural evolution share similar as well as diverging patterns.

Here, we focus on pattern similarities and examine the explanations given for their occurrence. Pattern similarity is found in how traits are distributed (through vertical descent with modification, horizontally and reticulately, or randomly through drift), and at what rate such distributions occur (gradually, by means of punctuated equilibria, cyclic, or periodically).

In what follows, we first define patterns as intermediary steps in evolutionary research that hold the middle between raw data and theoretical frameworks. Theoretical frameworks, in turn, refer to mechanisms to explain the patterns retrieved from modeling. Secondly, we demonstrate that pattern similarity is often explained from within the same theoretical frameworks and by referring to the same or similar evolutionary mechanisms. We investigate the validity of this assumption. Finally, we demonstrate that this analysis is worthwhile because it 
informs us on how linguistic and sociocultural evolution relate to overall biological evolution and on how we can identify multiple units, levels, mechanisms and processes of evolution.

\section{Defining Patterns}

Patterns emerge from how collected data is joined into descriptive or quantitative models of events. Scholars use methodological toolkits to generate the models, and the models bring forth observable patterns that in turn are explained by referring to theoretical frameworks. Patterns are thus intermediary steps in evolutionary research that hold the middle between raw data on the one hand and explanatory theoretical frameworks on the other (Gontier 2016; Grande \& Rieppel, 1994).

\subsection{Patterns are Not Raw Data}

Biological, linguistic and sociocultural evolution share similar patterns in (1) the directionality of trait distribution across lineages in time and/or space which can be vertical, horizontal, random, bi-or multi-directional, or cyclical; and (2) the rate whereby lineages evolve which can be gradual, fast, punctuated or periodic.

Points (1) and (2) provide a list of logical possibilities whereby we can respectively study how traits are distributed (the mode), or how we can conceptualize the rate whereby evolution occurs (the tempo), both at a micro- or macroscale (Simpson, 1944). Yet we are unfamiliar with thinking about patterns in this way. Instead, we have obtained our knowledge on the patterns of evolution through a historical learning process resulting from observing natural processes and testing hypotheses and theories on how evolution possibly occurs, as well as by experimenting how evolution can be adequately modeled (in scales of nature, timelines, trees, networks, cycles, etc.).

Patterns are informed by theories that make assumptions on how raw data should be ordered and represented. In addition, modeling by trees or networks nowadays often associates with a choice between different software packages that work from within different premises and heuristics.

Most evolutionary theories have so far focusses on how traits are distributed or transmitted across time and space, and at what rate such distribution has occurred, and that is the very reason why scholars have taken on the endeavor to model exactly these two aspects of the evolutionary process. We see this point more clearly by asking what other means there are to examine and model evolutionary phenomena.

One answer has been given by the ecological sciences that, rather than examining distribution (rates), examine how entities interact with each other and the abiotic world (Fox et al., 2001; Futuyma, 2010). Another answer has been given by evolutionary developmental schools that focus on how traits develop within individuals and groups (Hallgrimson \& Hall, 2011). Instead of studying 
phylogenetic splits, mergers or extinction, within evo-devo the emphasis lies on ontogenetic, intra-lineage phenomena. Still other means by which we can analyze evolution include hierarchy theory (Salthe, 1985), multilevel selection theory (Lewontin, 1970; Okasha, 2005) or major transitions (Maynard Smith \& Szathmáry, 1995).

\subsection{Patterns are Deduced from Theory-Informed Models and Diagrams}

Within macro-oriented phylogenetics, the mode and tempo of evolutionary phenomena are modeled either in rooted or unrooted and bi- or multifurcating trees or networks. Trees are often set in a Cartesian two-dimensional coordinate system where trait distribution is tracked linearly in space and over time. Tree models, therefore, work from within the premise of vertical, often hereditary transmission and they require time consistency. For those reasons, they cannot adequately depict instances of oblique, reversed, horizontal or multidirectional transmission (Feldman \& Cavalli-Sforza, 1976; Zhaxybayeva \& Doolittle, 2011).

These latter phenomena are modeled in networks. Networks are sometimes set in vector space, and they often remain unrooted because time-consistency is not always prioritized (Morrison, 2016). Nonetheless, the length and directionality of the branches sometimes indicate time as measured by the evolutionary distance between the modelled entities. Networks model all sorts of relationships occurring within and between biological, linguistic or cultural entities. And they do so not only in the past but also in the present, thereby assuming that ontogeny and ecology have relevant evolutionary roles to play.

Interactions and developmental processes are also represented by unrooted networks, circles or overlapping Venn diagrams that track cycles (Bechtel, 2011). Cycles are little-appreciated patterns. They can be found in circadian rhythms, gene-regulatory networks, protein folding, overall anatomical, sexual, cognitive, linguistic, and cultural development, and aging. Cycles differ from trees and networks because the latter two model events (speciation, extinction, merging or splitting of branches) while cycles model recurring and recursive processes. Cycles, moreover, often show stability over time, and they sometimes follow periodicities (either occurring at intervals or reoccurring at specific moments during ontogeny), but no diagrams exist yet to model these periodicities.

\section{Pattern Similarity and Evolutionary Frameworks}

Patterns are observed in diagrams and evolutionary models that are informed by theory. Theories underlie evolutionary frameworks, and the latter, in turn, refer to mechanisms to explain the patterns of evolution. Historically, the identification of evolutionary patterns associated with the introduction of different evolutionary theories on the mode and tempo of evolution, and with an investigation into which diagrams and models are best suited to order the data deemed relevant. For those reasons, similar patterns are explained by referring to the same evolutionary, often 
biological mechanisms. In this part, we investigate which patterns are associated with which theories and mechanisms.

\subsection{Pattern Similarity in Trait Distributions and Associated Mechanisms}

In the biological sciences, the study of vertical trait distribution associates with selection theory that demonstrates that gene distribution occurs via a pattern of descent with modification driven by heredity and positive selection of adaptive (genetic) traits (Dawkins, 1983). The preferred diagrams to model this pattern are trees and cladograms. Sociocultural and linguistic sciences have also found this pattern in how linguistic and cultural traits are transmitted across generations through (un)directed or biased learning and teaching. Consequently, the pattern similarity is explained by universal selection theories that are based upon the three Darwinian principles (Lewontin, 1970): differential variation, reproduction, and selection - that can also be understood as a recurring cycle (Cavalli-Sforza \& Feldman, 1981; Lumsden \& Wilson, 1981; Mesoudi, 2016).

However, vertical descent with modification can also result from random trait distributions, otherwise known as genetic drift. The founders of the Modern Synthesis recognized drift to alternate with natural selection, and especially Kimura (1960) explained how drift is a mechanism that occurs independently from natural selection. However, drift theory first emerged in linguistics (Greenberg, 1960; Jesperson, 1909; Koerper \& Stickel, 1980; Wittmann, 1969), and here, drift was often interpreted either as directed or as circular, and cyclic (Van Gelderen, 2013). Modern applications of drift theory are given by Bentley et al. (2004), Chang (2013), Centola et al. (2007), Chiaronia et al. (2009), and Koerper \& Stickel (1980).

Cycles associate with both macro-oriented disciplines such as ecology (e.g. the nitrogen cycle, that also relies on symbiosis) and micro-evolutionary research fields such as evolutionary developmental biology where scholars study intra- and intergenerational developmental processes. Cycles are also studied by linguistic and sociocultural scientists. Example include the cycle whereby children learn the language they speak from their community, while they also influence the language of the community and how it is taught to them (Mesoudi, 2016); or the cycle where biological evolution underlies the development of individual and group cognition that in turn influences cultural transmission whereafter the cycle repeats because cognition and cultural transmission can influence the course of biological evolution (Maynard Smith \& Szathmàry, 1995). Other examples include the iterated learning experiments and computational models that implement Bayesian statistics and Markov chain Monte Carlo simulations that mimic learning within and across generations (Briscoe, 1998; Tamariz \& Kirby, 2016). The outcome of such learning is depicted in tree models, but the learning process itself is cyclic and periodic, occurring at specific moments during development over multiple generations. Cycles currently remain unintegrated into 
phylogenetic representations, but for promising work on iterated learning networks, see Sole et al. (2010).

In biology, horizontal (from one lineage to another), bi- (between 2 lineages) and multi-directional (between multiple lineages) trait distribution has been associated with reticulate evolution as it occurs by means of lateral gene transfer, symbiogenesis, and hybridization (Arnold, 1997; Gontier, 2015; Keeling \& Palmer, 2008; Margulis, 1998; Zhaxybayeva \& Doolittle, 2011). In linguistic and sociocultural evolution, reticulations result from language and cultural borrowing, mixing, or hybridization (Croft, 2000; Shijulal et al., 2010; List, 2013). All these phenomena result in reticulate patterns which are better modeled by networks than trees. While especially the biological sciences have long downgraded the relevance of horizontal exchange, diachronic linguists and also anthropologists have always recognized the importance of reticulations in cultural diffusion studies.

\subsection{Pattern Similarity in Distribution Rates and Associated Mechanisms}

Natural selection theory traditionally predicts evolution to occur gradually. But the necessity of gradualness has been called into question by many scholars, including scholars that study punctuated equilibria (Gould \& Eldredge, 1977).

The pattern of punctuated equilibria recognizes two additional patterns that often occur sequentially in time. Long periods of stasis are intermitted by rapid periods of morphological change and/or speciation. The pattern can be detected at a molecular level (Pagel et al., 2006); as well as in the archeological record (Eldredge \& Tattersall, 1982), and in certain language families (Atkinson et al, 2008; Gray \& Jordan, 2000; Gray et al, 2009). But although the pattern can be found to occur repeatedly within all these different phenomena, it remains an open question whether punctuated equilibria also follow cycles or periodicities. If they do, then scholars should be able to uniformly clock and predict speciation/divergence and extinction/death rates, or how long recurring periods of stasis last. This, however, remains difficult regardless the fact that stasis, rapid speciation, and extinction occur repeatedly. It might also be unwarranted to assume that any uniformity can be found in how stasis or rapid change interchange one another. Until scholars can answer the current enigmas, punctuated equilibria are, therefore, best treated as a single pattern.

The rate of drift is difficult to calculate and differential depending upon the traits one examines to be drifting (genes or aspects of languages and cultures) (Cavalli-Sforza \& Feldman, 2003; Hallatschek et al., 2007; Nei et al., 1975). Drift can associate both with patterns of gradual descent with modification, or with punctuated equilibria. Over short time spans, drift often associates with a pattern similar to Brownian motion. Over longer periods of time, it either brings forth gradual patterns of change or patterns of stasis, i.e. periods wherein no reticulations or bifurcations of the lineages occur. This does not exclude the possibility for the lineage to change inside out through, for example, bottleneck 
or founder effects (that correlate with environmental circumstances and population size). When drift is followed by incremental growth, it brings forth more rapid, stage-like patterns.

Reticulate evolution often occurs at a fast evolutionary rate, because gene, trait or organismal (e.g. microbial) transfer, as well as sociocultural and linguistic transfer can occur a-sexually (without the requirement of reproducing a next generation). Nonetheless, in nature, obligate symbiotic partnerships can also constrain evolution and either result in co-evolution or stasis. And in culture, individual cognition or cultural learning and teaching also provides constraints and co-evolutionary dynamics of how languages and cultures evolve.

The same goes for the cycles studied by developmental evolutionary biologists, psychologists, and epigenetics (Hallgrimson \& Hall, 2011). Genes determine and constrain development thereby attributing to stability and stasis, but epigenetic changes can rapidly alter the course of ontogeny and phylogeny. Cycles are furthermore periodic, because they repeat over generations through time, and often at specific moments in time.

\section{Implications for How We Understand Linguistic and Cultural Evolution}

Similar patterns are often explained by the same specific biological mechanisms that subsequently become "universalized". But two problems arise. For one, assuming congruency between patterns and mechanisms is not always warranted because, as we saw in the previous part, the same patterns can sometimes be generated by different mechanisms. It follows that although tree and network models do lend insight into patterns of evolution, they do not straightforwardly demonstrate how, by which mechanisms, linguistic and cultural traits, organisms or species evolve. Secondly, when the same evolutionary theories are invoked to explain similar patterns, we find that the "universal" mechanisms held responsible become defined differentially within the different domains. Mechanismexplanations are often abandoned in favor of process accounts.

\subsection{Different Mechanisms Can Induce Similar Patterns}

While the pattern of descent with modification has spurred generations of researchers to investigate how bifurcation and extinction occur by means of natural selection, today we know that all mechanisms described above can bring forth this pattern. Descent with modification thus provides a general guideline to understand changing phenomena as undergoing evolution, but more research is required to examine how exactly this change occurs. Drift can, under certain circumstances, bring forth the pattern, but it can also bring forth patterns of punctuated equilibria. Reticulate evolutionary mechanisms bring forth reticulate patterns in a first phase, but in a second phase the reticulately acquired traits can undergo vertical descent with modification or bring forth patterns of co-evolution or stasis. 
The take-home messages of these findings are that (1) different mechanisms can bring forth the same patterns which implies that patterns are not clairvoyant identifiers of mechanisms whereby phenomena evolve; (2) no isomorphism between patterns and specific phenomena can be adhered to.

This makes it necessary to embrace a more pluralistic account on both the nature of patterns and mechanisms. Indeed, tracking the evolution of specific lineages over long periods of time often brings forth a sequential (perhaps sometimes cyclic) series of patterns, and a single phenomenon often evolves by multiple mechanisms.

Taking our hominin past as exemplar, we now have firm evidence that, in addition to evolving by means of natural selection, our species acquired genes through hybridization with other hominin species, and through lateral gene transfer with viruses and microorganisms. Rapid reticulate patterns are intermitted with gradual descent with modification, where mutated and acquired genes are transmitted vertically. In addition, our cultural and linguistic traits evolved through a combination of guided selection, random drift, and opportune reticulation.

\subsection{From Mechanism to Process Accounts}

The linguistic and sociocultural sciences have taken on an evolutionary outlook by looking for patterns and mechanisms in linguistic and cultural evolution that are similar to recognized patterns and mechanisms in the biological sciences. For those reasons, scholars have reformulated gene-based natural selection theory into universal Darwinian accounts. This has introduced a shift from investigating the mode and tempo of Darwin's mechanism of natural selection to identifying a variety of processes that are selective.

The same can be said about reticulations. Reticulate evolution does not always require lateral gene transfer or hybridization. Reticulations also characterize processes of language and cultural borrowing or the formation of multicultural societies. This again brings forth a shift from mechanism to process accounts, and the similarity is found in patterns rather than in mechanisms that are specific to genetic, physiological, developmental, linguistic or sociocultural evolution.

Scholars remain divided on how sociocultural and linguistic evolution relate to biological evolution. The analysis provided here shows that a universal evolutionary approach is possible if we recognize that different processes can causally lead to pattern similarity in life, language, and culture, even without fixed mechanisms. This implies that we need to go beyond theorizing on the nature of specific mechanisms and instead focus on the wide variety of processes whereby evolution occurs because these are differential. 


\section{Toward Unit, Level, and Mechanism Plurality}

Finally, the shift from mechanism to process accounts also alters how we define the units and levels of evolution, or more generally, how we define information. What counts as "information" in the biological, sociocultural and linguistic sciences and how "information transfer" is conceptualized is defined differentially by the different domains. These differences can also be taken as point of analysis to distinguish amongst research schools.

The gene is classically assumed to be the unit of information as well as the unit of selection. Scholars that want to reduce culture and language to biology have theorized that linguistic and sociocultural evolution can be reduced to the study of genes. Those that want to differentiate language and culture from biological evolution have often pointed out that more than genes are exchanged in linguistic and cultural evolution. And those that want to understand language, culture and biology as co-evolving realms have on the one hand searched for cultural replicators such as memes that perform functions similar to genes (Dawkins, 1983), and on the other, they have searched for other units of evolution, that surpass the scope of replicators, such as interactors (Hull, 2001) and reproducers (Griesemer, 2000).

These debates have mostly been held at a theoretical level. They do not stroke well with actual scientific practices where scholars study the transfer of a wide variety of phenomena that are understood, not only to carry information, but also to causally inflict change.

The information that linguists track extends classic etymological research and involves linguistic traits such as cognates, loan words and doublets, or linguistic universals including syntax typology (e.g. the transitions from SOV to SVO). Sociocultural scholars draw their phylogenies and networks of sociocultural evolution by tracking material artifacts ranging from paleolithic stone tools to modern skate board decks (Prentiss et al., 2016), or immaterial mentifacts such as fairy tales (da Silva \& Tehrani, 2016) or religious ideas. And while biologists used to focus on the differential distribution and transmission of morphological traits amongst organisms, species and higher taxa over geological time, today, they focus more on the divergence of genes and proteins over time, which they calculate by making use of (relaxed) molecular clock models.

All domains are moving toward a general recognition that evolution, be it biological, social or linguistic in kind, occurs through a myriad of units at various levels of an ontological hierarchy, and by numerous processes rather than by a fixed set of theorized mechanisms. It are the interactions amongst the units and levels that induce evolutionary change and that bring forth pattern similarity.

\section{Acknowledgements}

Written with the support of the Portuguese Fund for Science and Technology, grant ID SFRH/BPD/89195/2012 and project number UID/FIL/00678/2013. 


\section{References}

Arnold, M. L. (1997). Natural hybridization and evolution. New York, NY: Oxford University Press.

Atkinson, Q. D., Meade, A., Venditti, C., Greenhill, S. \& Pagel, M. (2008). Languages evolve in punctuational bursts. Science, 319, 588.

Bechtel, W. (2011). Mechanism and biological explanation. Philosophy of Science, 78, 533-577.

Bentley, R., Hahn, M. \& Shennan, S. (2004). Random drift and culture change. Proceedings of the Royal Society B, 271, 1443-1450.

Briscoe, E. J. (1998). Language as a complex adaptive system: Coevolution of language and of the language acquisition device. In: van Halteren, H., et al. (Eds.), Proceedings of eighth computational linguistics in the Netherlands Conference (pp. 3-40). The Netherlands, Amsterdam.

Cavalli-Sforza, L. L. \& Feldman, M. W. (1981). Cultural transmission and evolution. Princeton, NJ: Princeton University Press.

Cavalli-Sforza, L. L., Feldman, M. W. (2003). The application of molecular genetic approaches to the study of human evolution. Nature Genetics, 33, 26675.

Centola, D., González-Avella, J., Eguíluz, V. \& San Miguel, M. (2007). Homophily, cultural drift, and the co-evolution of cultural groups. Journal of Conflict Resolution, 51, 905-929.

Chang, C. (2013). A novelty effect in phonetic drift of the native language. Journal of Phonetics, 41, 520-33.

Chiaronia, J., Underhillb, P. \& Cavalli-Sforza, L. (2009). Y chromosome diversity, human expansion, drift, and cultural evolution. Proceedings of the National Academy of Science, USA, 106, 20174-20179.

Croft, W. (2000). Explaining language change: An evolutionary approach. Essex, Pearson.

da Silva, S. G., Tehrani, J. J. (2016). Comparative phylogenetic analyses uncover the ancient roots of Indo-European folktales. Royal Society Open Science, 3, DOI: $10.1098 /$ rsos. 150645 .

Dawkins, R. (1983). Universal Darwinism. In Hull, D.L. \& Ruse, M. (Eds.), The philosophy of biology (pp. 15-35). New York, NY: Oxford University Press.

Eldredge, N. \& Tattersall, I. (1982). The myths of human evolution. New York, NY, Columbia University Press.

Feldman, M. \& Cavalli-Sforza, L. (1976). Cultural and biological evolutionary processes, selection for a trait under complex transmission. Theoretical Population Biology, 9, 238-59.

Fox, C. W., Roff, D. A. \& Fairbairn, D. J. (2001). Evolutionary Ecology: Concepts and case studies. Oxford, Oxford University Press.

Futuyma, D. J. (2010). Evolutionary constraint and ecological consequences. Evolution, 64, 1865-1884. 
Gontier, N. (2016). Guest-Editorial Introduction: Converging Evolutionary Patterns in Life and Culture. Evolutionary Biology, 43 (4), 427-445.

Gontier, N. (Ed.) (2015). Reticulate Evolution. Dordrecht, Springer.

Gould, S. J., Eldredge, N. (1977). Punctuated equilibria: The tempo and mode of evolution reconsidered. Paleobiology, 3, 115-151.

Grande, L. \& Rieppel, O., (Eds.). (1994). Interpreting the hierarchy of nature. San Diego, CA, Academia Press.

Gray, R. D. \& Jordan, F. M. (2000). Language trees support the express-train sequence of Austronesian expansion. Nature, 405, 1052-1055.

Gray, R. D., Drummond, A. J. \& Greenhill, S. J. (2009). Language phylogenies reveal expansion pulses and pauses in Pacific settlement. Science, 323, 479483.

Greenberg, J. H. (1960). A quantitative approach to the morphological typology of language. International Journal of American Linguistics, 26, 178-94.

Griesemer, J. (2000). The units of evolutionary transition. Selection, 1, 67-80.

Hallatschek, O., Hersen, P., Ramanathan, S., Nelson, D. R. (2007). Genetic drift at expanding frontiers promotes gene segregation. Proceedings of the National Academy of Sciences, USA, 104 (50), 19926-19930.

Hallgrimsson, B. \& Hall, B. (Eds.) (2011). Epigenetics. Berkeley, CA: University of California Press.

Hull, D. L. (2001). Science and Selection. New York, Cambridge University Press.

Jespersen, O. (1909). A modern English grammar on historical principles. London: Allen \& Unwin.

Keeling, P. J. \& Palmer, J. D. (2008). Horizontal gene transfer in eukaryotic evolution. Nature Reviews, Genetics, 9, 605-618.

Kimura, M. (1968). Evolutionary rate at the molecular level. Nature, 217, 624626.

Koerper, H., Stickel, E. (1980). Cultural drift: A primary process of culture change. Journal of Anthropological Research, 36, 463-469.

Krause, J., et al. (2007). The derived FOXP2 variant of modern humans was shared with Neanderthals. Current Biology, 17, 1908-12.

Lewontin, R. (1970). The levels of selection. Annual Review of Ecological Systems, 1, 1-18.

List, J.-M., et al. (2013). Networks of lexical borrowing and lateral gene transfer in language and genome evolution. Bioessays, 36, 141-150.

Lumsden, C. \& Wilson, E. (1981). Genes, mind and culture: The coevolutionary process. Cambridge, MA: Harvard University Press.

Margulis, L. (1998). The symbiotic planet. London: Phoenix Orion Books.

Maynard Smith, J. \& Szathmáry, E. (1995). The major transitions in evolution. New York, NY: Oxford University Press.

Mesoudi, A. (2016). Cultural evolution: A review of theory, findings and controversies. Evolutionary biology, 43 (4), 481-497. 
Morrison, D. (2016). Genealogies: Pedigrees and phylogenies are reticulating networks, not just diverging trees. Evolutionary Biology, 43, 456-473.

Nei, M., Maruyama, T., Chakraborty, R. (1975). The bottleneck effect and genetic variability in populations. Evolution, 29, 1-10.

Okasha, S. (2005). Multilevel selection and the major transitions in evolution. Philosophy of Science, 72, 1013-1025.

Pagel, M. (1999). Inferring the historical patterns of biological evolution. Science, 401, 877-884.

Pagel, M., Venditti, C., Meade, A. (2006). Large punctuational contribution of speciation to evolutionary divergence at the molecular level. Science, 314, 119-121.

Prentiss, A. M., Walsh, M. J., Skelton, R. R., Mattes, M. (2016) Mosaic evolution in cultural frameworks: Skateboard decks and projectile points. In Medoza Straffon, L. (Ed.), Cultural phylogenetics (pp. 113-130). Dordrecht, Springer Nature.

Rali, F., \& Griffiths, T. (2010). Words as alleles: Connecting language evolution with Bayesian learners to models of genetic drift. Proceedings of the Royal Society of London B, 277, 429-436.

Salthe, S. (1985). Evolving hierarchical systems: Their structure and representation. New York: Columbia University Press.

Shijulal, N. S., et al. (2010). Networks uncover hidden lexical borrowing in IndoEuropean language evolution. Proceedings of the Royal Society B, doi:10.1098/rspb.2010.1917.

Simpson, G. G. (1944). Tempo and mode in evolution. New York, NY, Columbia University Press.

Sole, R. V., et al. (2010). Language networks: Their structure, function, and evolution. Complexity, 15, 20-26.

Steels, L. (2015). The talking heads experiment: Origins of words and meanings. Berlin, Language Science Press.

Tamariz, M. \& Kirby, S. (2016). The cultural evolution of language. Current Opinion in Psychology, 8, 37-43.

Van Gelderen, E. (2013). The linguistic cycle and the language faculty. Language and Linguistics Compass, 7, 233-250.

Wittmann, H. (1969). The Indo-European drift and the position of Hittite. International Journal of American Linguistics, 35, 266-68.

Zhaxybayeva, O., \& Doolittle, W. F. (2011). Lateral gene transfer. Current Biology, 21(7), R242-R246. 